\section{Successful treatment of Leuconostoc bacteremia in a neutropenic patient with tigecycline}

\author{
Trupti Patel,' Aoife Molloy, ${ }^{2}$ \\ Robin Smith, ${ }^{1}$ Indran Balakrishnan ${ }^{1}$ \\ 'Department of Microbiology, Royal Free \\ Hospital NHS Foundation Trust, London; \\ 2Department of Infectious Diseases, \\ Ealing Hospital, Ealing Hospital NHS \\ Trust, Southall, UK
}

\section{Abstract}

Leuconostoc lactis is a recognised cause of infection in immunocompromised hosts. It is intrinsically resistant to multiple antibiotics and treatment options may be limited. We report a case of safe and effective use of tigecycline in the treatment of Leuconostoc catheter-related line sepsis in a neutropenic patient. To our knowledge, this is the first reported case of successful use of tigecycline for Leuconostoc bacteremia.

\section{Case Report}

A 52-year-old woman with granulocytic sarcoma affecting the anterior cranial fossa and frontal sinus was commenced on ADE (cytarabine, daunorubicin and etoposide) and Mylotarg (gemtuzumab) through a peripherally inserted central catheter (PICC) line as part of the AML 17 trial (http://aml17.cardiff.ac.uk /aml17/Default.aspx). She was known to have a beta-lactam allergy manifest as an erythematous rash.

On day 9 of chemotherapy, meropenem was empirically started for febrile neutropenia as per fever and neutropenia guidelines. ${ }^{1}$ Her fever resolved after 48 hours and meropenem was stopped after 7 days. On day 18 of chemotherapy she was still neutropenic and developed further fevers to $39^{\circ} \mathrm{C}$. Her PICC insertion site appeared erythematous with an associated blister. Meropenem was restarted in addition to teicoplanin. She remained febrile over the next three days and caspofungin was started in view of high-resolution computed topography (HRCT) chest findings compatible with possible fungal infection and a positive initial serum galactomannan assay (although the repeat specimen was negative). After 10 days of treatment she developed a rash and continued to spike temperatures. Meropenem was therefore replaced with ciprofloxacin as it was thought to be responsi- ble for the rash. All cultures including multiple blood cultures and PICC line site swabs were culture negative.

On day 31 of chemotherapy she was still febrile and blood cultures from peripheral veins and the PICC line taken on day 30 yielded gram-positive cocci in pairs and chains after 24 and 11 hours' culture respectively. The isolate was identified as Leuconostoc lactis using the BD Phoenix identification (Becton, Dickinson and Company, USA) and $\mathrm{API}^{\circledR}$ rapid ID 32 Strep systems (bioMérieux, France). Mean inhibitory concentrations (MICs) determined by E-test (AB Biodisk, Sweden) were: penicillin $0.5 \mathrm{mg} / \mathrm{L}$, vancomycin $>256 \mathrm{mg} / \mathrm{L}$, teicoplanin $256 \mathrm{mg} / \mathrm{L}$, ciprofloxacin $1.0 \mathrm{mg} / \mathrm{L}$, tigecycline $0.064 \mathrm{mg} / \mathrm{L}$, linezolid $1.5 \mathrm{mg} / \mathrm{L}$ and daptomycin $0.064 \mathrm{mg} / \mathrm{L}$. On day 32 of chemotherapy, ciprofloxacin and teicoplanin were changed to intravenous linezolid and the PICC was removed. The next day therapy was changed to tigecycline (50 mg IV q12h) because of concerns over myelotoxicity (anaemia and thrombocytopenia) associated with linezolid. ${ }^{2}$ Daptomycin was unavailable in the hospital formulary and ampicillin was not used due to concerns over beta-lactam allergy and a borderline penicillin E-test MIC. Culture of the PICC tip yielded no significant growth. The patient continued to have low-grade fevers over the next four days, despite a negative transthoracic echocardiogram. On the fourth day of treatment, however, her fever resolved and she was discharged from hospital after completing 8 days of tigecycline, to which she had no adverse effects. She remained asymptomatic with sterile blood cultures and successfully continued her chemotherapy.

\section{Discussion}

Leuconostoc spp. are catalase-negative, gram-positive, facultatively anaerobic coccobacilli. They are environmental organisms often found on plants, dairy products, vegetables, wine and occasionally in human vaginal and stool samples. ${ }^{3}$ Although an uncommon human pathogen, cases of bacteremia, endocarditis, pneumonia, meningitis, osteomye litis, peritonitis, brain and liver abscesses have been described. . $^{4-12}$

Leuconostoc spp. and other gram-positive antimicrobial-resistant organisms are increasingly recognised as important pathogens in neutropenic patients probably due, in addition to immunosuppression, to the use of indwelling intravascular devices, antibiotic prophylaxis and evolution of chemotherapeutic agents. ${ }^{13}$ Infection with Leuconostoc may cause fever, intravenous catheter-related sepsis, bacteremia, abdominal pain, gastroenteritis, colitis or meningitis in this group of patients.
Correspondence: Trupti Patel, Department of Microbiology, Royal Free Hospital NHS Foundation Trust, Pond Street, London NW3 2QG, UK. Tel. +44.207.794.0500

E-mail: truptipatel1@nhs.net

Key words: Leuconostoc, tigecycline, bacteremia, immunocompromise, neutropenia.

Contributions: TP, AM, contributed to manuscript preparation; RS, IB, were involved in reviewing the manuscript.

Conflict of interests: the authors report no conflict of interests.

Received for publication: 31 January 2011.

Revision received: 12 March 2012.

Accepted for publication: 17 March 2012.

This work is licensed under a Creative Commons Attribution NonCommercial 3.0 License (CC BYNC 3.0).

(C) Copyright T. Patel et al., 2012

Licensee PAGEPress, Italy

Infectious Disease Reports 2012; 4:e31

doi:10.4081/idr.2012.e31

Other reported risk factors for infection include a history of surgery and prior vancomycin therapy. ${ }^{7,14}$ Common portals of entry described include intravascular catheters or the gastrointestinal tract. ${ }^{15,16}$ Removal of intravenous catheters alone has been shown to be curative in some patients without the need for antimicrobial therapy. ${ }^{7}$

There are no standardised criteria for interpreting the antimicrobial susceptibility testing of Leuconostoc spp. - therapy should be guided by the MIC of the isolate. Leuconostoc spp. are intrinsically resistant to glycopeptides, owing to the production of peptidoglycan precursors ending in D-Ala-D-Lac, but are usually susceptible to penicillin, ampicillin, aminoglycosides, clindamycin, minocycline and macrolides. ${ }^{17}$ In addition, linezolid and daptomycin have been used successfully to treat Leuconostoc bacteremia, although linezolid MICs of Leuconostoc spp. are usually higher when compared with those of streptococci. ${ }^{3,18}$ Moderate susceptibility is seen with cephalosporins, chloramphenicol, tetracycline and doxycycline. ${ }^{3}$ Although the organism has been shown to be resistant to cefoxitin, it is susceptible to cefotaxime in vitro. ${ }^{19}$ This may have been an alternative therapeutic option in our patient.

Tigecycline, a glycylcycline, is a broad spectrum synthetic derivative of minocycline which has a broad spectrum of activity against various gram-positive and gram-negative bacteria including multidrug-resistant strains, anaerobic bacteria and atypical organisms. It has proven to be useful in the treatment of hospi- 
tal-acquired infections caused by vancomycinintermediate and vancomycin-resistant enterococci (VRE), meticillin-resistant Staphylococcus aureus (MRSA), extended-spectrum $\beta$-lactamase (ESBL)-producing Enterobacteriaceae, multidrug-resistant Acinetobacter baumanii and penicillin-resistant Streptococcus pneumoniae..$^{20,21}$

In the UK, its licensed indications are complicated intra-abdominal and complicated skin and soft tissue infection. ${ }^{22,23}$ It is also licensed for the treatment of community-acquired pneumonia in the US. ${ }^{24}$

\section{Conclusions}

Although tigecycline has been demonstrated to be a safe and effective second-line option in microbiologically documented infections in neutropenic patients, there have been no trials to determine whether tigecycline is effective in neutropenic bacteremia and there are also theoretical concerns surrounding low serum concentrations (due to a large volume of distribution) and its mostly bacteriostatic activity. ${ }^{25}$ It is for this reason that tigecycline is not generally recommended for primary bacteremia but it is used for secondary bacteremia associated with complicated skin and soft tissue infections, intra-abdominal infections and community-acquired pneumonia. ${ }^{26}$ Despite these concerns, here we report the first successful use of tigecycline in the treatment of Leuconostoc bacteremia in a neutropenic patient.

\section{References}

1. Freifeld AG, Bow EJ, Sepkowitz KA, et al. Clinical practice guideline for the use of antimicrobial agents in neutropenic patients with cancer: 2010 update by the infectious diseases society of america. Clin Infect Dis 2011;52:e56-93.

2. Bernstein WB, Trotta RF, Rector JT, et al. Mechanisms for linezolid-induced anemia and thrombocytopenia. Ann Pharmacother 2003;37:517-20.

3. Arias CA, Murray BE. Enterococcus species, Streptococcus bovis group and Leuconostoc species. In: Mandell GL, Bennett JE, Dolin R. Mandell, Douglas, and Bennett's principles and practice of infec- tious diseases, 7th edn. Philadelphia: Churchill Livingstone/Elsevier; 2009.

4. Albanese A, Spanu T, Sali M, et al. Molecular identification of Leuconostoc mesenteroides as a cause of brain abscess in an immunocompromised patient. J Clin Microbiol 2006;44:3044-5.

5. Buu-Hoi A, Branger C, Acar JF. Vancomycin-resistant streptococci or Leuconostoc sp. Antimicrob Agents Chemother 1985;28:458-60.

6. Coovadia YM, Solwa Z, van Den EJ. Potential pathogenicity of Leuconostoc. Lancet 1988;1:306.

7. Handwerger S, Horowitz H, Coburn K, et al. Infection due to Leuconostoc species: six cases and review. Rev Infect Dis 1990;12:602-10.

8. Ishiyama K, Yamazaki H, Senda Y, et al. Leuconostoc bacteremia in three patients with malignancies. J Infect Chemother 2011;17:412-8.

9. Kocak F, Yurtseven N, Aydemir N, et al. A case of osteomyelitis due to Leuconostoc lactis. Scand J Infect Dis 2007;39:278-80.

10. Templin KS, Crook T, Riley T III, et al. Spontaneous bacterial peritonitis and bacteremia due to Leuconostoc species in a patient with end-stage liver disease: a case report. J Infect 2001;43:155-7.

11. Vagiakou-Voudris E, Mylona-Petropoulou D, Kalogeropoulou E, et al. Multiple liver abscesses associated with bacteremia due to Leuconostoc lactis. Scand J Infect Dis 2002;34:766-7.

12. Yamazaki R, Mori T, Sugita K, et al. Leuconostoc septicemia in a neutropenic patient with acute myelogenous leukemia relapsed after allogeneic peripheral blood stem cell transplantation. Transpl Infect Dis 2009;11:94-5.

13. Zinner SH. Changing epidemiology of infections in patients with neutropenia and cancer: emphasis on gram-positive and resistant bacteria. Clin Infect Dis 1999;29:490-4.

14. Montejo M, Grande C, Valdivieso A, et al. Abdominal abscess due to leuconostoc species in a liver transplant recipient. $\mathrm{J}$ Infect 2000;41:197-8.

15. Florescu D, Hill L, Sudan D, Iwen PC. Leuconostoc bacteremia in pediatric patients with short bowel syndrome: case series and review. Pediatr Infect Dis J 2008;27:1013-9.

16. Lee MR, Huang YT, Lee PI, et al. Healthcare-associated bacteraemia cau sed by Leuconostoc species at a university hospital in Taiwan between 1995 and 2008 . J Hosp Infect 2011;78:45-9.

17. Swenson JM, Facklam RR, Thornsberry C. Antimicrobial susceptibility of vancomycin-resistant Leuconostoc, Pediococcus, and Lactobacillus species. Antimicrob Agents Chemother 1990;34: 543-9.

18. Golan Y, Poutsiaka DD, Tozzi S, et al. Daptomycin for line-related Leuconostoc bacteraemia. J Antimicrob Chemother 2001;47:364-5.

19. de la Maza L, Ruoff KL, Ferraro MJ. In vitro activities of daptomycin and other antimicrobial agents against vancomycin-resistant gram-positive bacteria. Antimicrob Agents Chemother 1989;33:1383-4.

20. Townsend ML, Pound MW, Drew RH. Tigecycline: a new glycylcycline antimicrobial. Int J Clin Pract 2006;60:1662-72.

21. Norskov-Lauritsen N, Marchandin H, Dowzicky MJ. Antimicrobial susceptibility of tigecycline and comparators against bacterial isolates collected as part of the TEST study in Europe (2004-2007). Int J Antimicrob Agents 2009;34:121-30.

22. Babinchak T, Ellis-Grosse E, Dartois N, et al. The efficacy and safety of tigecycline for the treatment of complicated intraabdominal infections: analysis of pooled clinical trial data. Clin Infect Dis 2005;41 Suppl5:S354-67.

23. Ellis-Grosse EJ, Babinchak T, Dartois N, et al. The efficacy and safety of tigecycline in the treatment of skin and skin-structure infections: results of 2 double-blind phase 3 comparison studies with vancomycinaztreonam. Clin Infect Dis 2005;41 Suppl 5:S341-53.

24. Bergallo C, Jasovich A, Teglia 0, et al. Safety and efficacy of intravenous tigecycline in treatment of community-acquired pneumonia: results from a double-blind randomized phase 3 comparison study with levofloxacin. Diagn Microbiol Infect Dis 2009;63:52-61.

25. Chemaly RF, Hanmod SS, Jiang Y, et al. Tigecycline use in cancer patients with serious infections: a report on 110 cases from a single institution. Medicine (Baltimore) 2009;88:211-20.

26. Gardiner D, Dukart G, Cooper A, Babinchak T. Safety and efficacy of intravenous tigecycline in subjects with secondary bacteremia: pooled results from 8 phase III clinical trials. Clin Infect Dis 2010;50:229-38. 